\title{
COMMUNICATIONS in PHYSICS
}

Volume 27, Number 3

September 2017

\section{PAPERS}

Yield Ratios of the Isomeric Pair ${ }^{179 m, g} \mathbf{W}$ Produced in the ${ }^{n a t} \mathbf{W}(\mathrm{g}, \mathbf{x n}){ }^{179 m, g} \mathbf{W}$ Reactions with 50-65 MeV Bremsstrahlung

Nguyen Van Do, Pham Duc Khue, Nguyen Thi Xuan, Bui Van Loat, Nguyen Thi Hien and Guinyun Kim

Radiation Dose Estimation of Cement Samples Used in Lao PDR

Sonexay Xayheungsy, Le Hong Khiem and Le Dai Nam

Edge Effects of Truncated Dirac Solitons in Binary Waveguide Arrays

Tran Xuan Truong, Nguyen Minh Hue and Phung Dinh Hoat

Population Distribution of Vibrational Levels of the $2^{1} \Pi$ State of NaLi

Fabrication of Electrochemical Electrodes Based on Platinum and ZnO

Nanofibers for Biosensing Applications

Dang Thi Thanh Le, Nguyen Van Hoang, Nguyen Van Hieu, Vu Quang Khue and Tran Quang Huy

Fabrication and Evaluation of the Photocatalytic, Antibacterial Activity of $\mathrm{AgTiO}_{2}$ Thin Film

Nu Quynh Trang Ton, Thi Ngoc Tu Le, Dang Trai Nguyen and Thi Hanh Thu Vu

Preparation of NiPt Alloys by Galvanic Replacement Reaction on Ni Films for Direct Ethanol Fuel Cell

Pham Van Vinh

Fabrication and Magnetic Properties of Sintered $\mathrm{SrFe}_{12} \mathrm{O}_{19}-\mathrm{NiFe}_{2} \mathrm{O}_{4}$

Tran Thi Viet Nga and To Thanh Loan

Relaxation Rate and Mobility of a Two-dimensional Electron Gas in $\mathrm{MgZnO} / \mathrm{ZnO}$ Heterostructures Including Exchange and Correlation Effects Vo Van Tai and Nguyen Quoc Khanh 
